\title{
A NOTE ON NUMERABLE COVERS
}

\section{J. DERWENT}

1. Introduction. In [2], A. Dold has introduced and used to great advantage the notion of numerable cover, i.e., a cover that is refined by a partition of unity. In this note we give in $\$ 3$ several equivalent forms of this notion. They may be viewed as giving simultaneously generalizations and new proofs of several of the theorems in [3, pp. 156-160]. In $\$ 4$ we use one of them to give a short proof of the section extension theorem for numerable fiber bundles with contractible fiber.

2. Notation. We generally follow Kelley [3] and Dold [2]. Let $\mathfrak{U}=\left\{U_{\alpha}: \alpha \in A\right\}$ be a cover of a topological space $X$. Let $\mathfrak{Z}$ $=\left\{p_{\beta}: \beta \in B\right\}$ be a family of continuous functions on $X$ with values in the unit interval $[0,1]$. The support of $p_{\beta}$ is $V_{\beta}^{-}$(the bar indicates closure) where $V_{\beta}=\left\{x: p_{\beta}(x)>0\right\}$. We attribute to $\mathfrak{Y}$ properties of the collection $\left\{V_{\beta}: \beta \in B\right\}$. In particular $\mathfrak{V}$ is $\sigma$-discrete if $B$ $=\bigcup\left\{B_{n}: n=1,2, \cdots\right\}$, a disjoint union such that $\left\{V_{\beta}: \beta \in B_{n}\right\}$ is discrete for each $n$. We say that $\mathfrak{U}$ is numerable if there is a locally finite partition of unity which refines $\mathfrak{U}$, and that $\mathfrak{U}$ is $\sigma$-numerable if there is a family $\mathfrak{V}=\left\{p_{\beta}: \beta \in B_{n}, n=1,2, \cdots\right\}$ which refines $\mathfrak{U}$ such that $\left\{p_{\beta}: \beta \in B_{n}\right\}$ is locally finite for each $n$.

3. Theorem. For a cover $\mathfrak{U}=\left\{U_{\alpha}: \alpha \in A\right\}$ of $X$, the following are equivalent:

(i) $\mathfrak{U}$ is numerable,

(ii) there is a locally finite partition of unity $\left\{p_{\alpha}: \alpha \in A\right\}$ such that $\left\{x: p_{\alpha}(x)>0\right\} \subset U_{\alpha}$ for each $\alpha \in A$; (some $p_{\alpha}$ 's may be identically 0 ),

(iii) there is a locally finite partition of unity whose supports refine $\mathfrak{u}$ and which is $\sigma$-discrete,

(iv) $\mathfrak{U}$ is $\sigma$-numerable.

Moreover, in (iii), if each point of $X$ belongs to at most $m$ members of $\mathfrak{U}$, then we may choose $B_{n}=\varnothing$ for $n>m$.

Proof. (i) $\Rightarrow$ (ii). Let $\left\{q_{\beta}: \beta \in B\right\}$ be a locally finite partition of unity refining $\mathfrak{U}$. Let $\tau: B \rightarrow A$ be such that $\left\{x: q_{\beta}(x)>0\right\} \subset U_{\tau(\beta)}$ for $\beta \in B$. Let $p_{\alpha}(x)=0$ if $\tau^{-1}(\alpha)=\varnothing$. Otherwise

$$
p_{\alpha}(x)=\sum\left\{q_{\beta}(x): \tau(\beta)=\alpha\right\} .
$$

(ii) $\Rightarrow$ (iii). The statement in (iii) was inspired by [1, Lemma 1$]$.

Received by the editors May 29, 1967. 
The proof is based on [4, pp. 25-26] and [5, p. 192]. Let $B$ be the family of all nonempty finite subsets of $A$. If $\beta \in B$, $\# \beta$ is the number of elements in $\beta$. We will have $B_{n}=\{\beta \in B: \# \beta=n\}$. For $\beta \in B$ define

$$
\begin{aligned}
m_{\beta}(x) & =\min \left\{p_{\alpha}(x): \alpha \in \beta\right\}, \quad M_{\beta}(x)=\max \left\{p_{\alpha}(x): \alpha \notin \beta\right\}, \\
P(x) & =\max \left\{0, m_{\beta}(x)-M_{\beta}(x): \beta \in B\right\}>0 \text { and } \\
Q_{\beta}(x) & =\max \left\{0, m_{\beta}(x)-M_{\beta}(x)-\frac{1}{2} P(x)\right\} .
\end{aligned}
$$

It is our contention that

(1) if $V_{\beta}=\left\{x: Q_{\beta}(x)>0\right\}$ and $W_{\beta}=\left\{x: m_{\beta}(x)>M_{\beta}(x)\right\}$, then $V_{\beta}^{-} \subset W_{\beta} \subset \cap\left\{U_{\alpha}: \alpha \in \beta\right\}$;

(2) $\left\{V_{\beta}: \beta \in B\right\}$ is a locally finite cover of $X$;

(3) $\left\{V_{\boldsymbol{\beta}}^{-}: \beta \in B_{n}\right\}$ is discrete.

The second containment in (1) is obvious from the definition of $m_{\beta}$ and $M_{\beta}$. On the other hand if $x \in V_{\beta}^{-}$then $m_{\beta}(x)-M_{\beta}(x) \geqq \frac{1}{2} P(x)>0$ and so $x \in W_{\beta}$.

For (2) let $x \in X$ and let $V$ be a neighborhood of $x$ such that $\gamma$ $=\left\{\alpha \in A: V \cap U_{\alpha} \neq \varnothing\right\}$ is finite. If $V \cap V_{\beta} \neq \varnothing$ then, by (1), $\beta \subset \gamma$. Furthermore, if $P(x)=m_{\beta}(x)-M_{\beta}(x)$ then $Q_{\beta}(x)>0$.

To prove (3) it is sufficient to observe with Milnor [4, p. 26] that $\left\{W_{\beta}: \beta \in B_{n}\right\}$ is pairwise disjoint.

Finally let $q_{\beta}=Q_{\beta} / \sum\left\{Q_{\beta^{\prime}}: \beta^{\prime} \in B\right\}$.

(iii) $\Rightarrow$ (iv). Obvious.

(iv) $\Rightarrow$ (i). This is due to Dold [2, proof on the top half of page 237].

The last statement is a consequence of (1) in the proof that (ii) $\Rightarrow$ (iii).

Corollary. Let $p: E \rightarrow B$ be a fibration which is trivial over each set of a numerable cover of $B$. Then there is a countable locally finite partition of unity $\left\{p_{n}: n=1,2, \cdots\right\}$ such that $E$ is trivial on a neighborhood of the support of $p_{n}$ for each $n$.

Proof. Apply (iii) and let $p_{n}=\sum\left\{q_{\beta}: \beta \in B_{n}\right\}$.

4. The section extension property. The theorem we prove in this section is a consequence of a more general theorem of Dold [2, p. 229]. We include it because of its simplicity and to illustrate the use of the corollary above. We say that $V$ is a halo of $A$ in $B$ if there is a map $f: B \rightarrow[0,1]$ such that $A \subset f^{-1}(0)$ and $B-V \subset f^{-1}(1)$.

Lemma. Let $p: E \rightarrow B$ be a fiber bundle with contractible fiber $F$. Let $V$ be a halo of $A$ in $B$ and let $s: V \rightarrow E$ be a section over $V$. If $E$ is trivial over $U$, there is a halo $W$ of $A$ in $B$ and a section $s: W \cup U \rightarrow E$ such that $W \subset V$ and $S|W=s| W$. 
Proof. Let $f: B \rightarrow[0,1]$ be such that $A \subset f^{-1}(0)$ and $B-V \subset f^{-1}(1)$. Let $\phi: U \times F \rightarrow E$ be a trivialization over $U$. Let $H: F \times I \rightarrow F$ be such that $H(x, 0)=x$ and $H(x, 1)=*$. We set $W=\left\{b \in B: f(b)<\frac{1}{2}\right\}$. Define $\tilde{s}: V \cap U \rightarrow F$ by $\phi^{-1}(s(b))=(b, \tilde{s}(b))$. Finally define $S: W \cup U \rightarrow E$ by

$$
\begin{aligned}
S(b) & =s(b) \text { if } b \in W \text { and } f(b) \leqq \frac{1}{2}, \\
& =\phi\left(b, H(\tilde{s}(b), 4 f(b)-2) \text { if } b \in V \cap U \text { and } \frac{1}{2} \leqq f(b) \leqq \frac{3}{4},\right. \\
& =\phi(b, *) \text { if } b \in U \text { and } f(b) \geqq \frac{3}{4} .
\end{aligned}
$$

THEOREM. Let $p: E \rightarrow B$ be a numerable fiber bundle with contractible fiber $F$. Let $V$ be a halo of $A$ in $B$ and let $s: V \rightarrow E$ be a section over $E$. Then there is a section $S: B \rightarrow E$ such that $S|A=s| A$.

Proof. Let $\left\{p_{n}: n=1,2, \cdots\right\}$ be a partition of unity as in the corollary above, with $E$ trivial on a neighborhood $U_{n}$ of the support of $p_{n}$ for each $n$. Let $f_{n}=1-p_{1}-p_{2}-\cdots-p_{n}$. Let $B_{n}=f_{n}^{-1}(0)$ and $A_{n}=A \cup B_{n}$. Applying the lemma to $V, A$ and $U_{1}$, we get a section $s_{1}: W_{1} \cup U_{1} \rightarrow W$ where $W_{1}$ is a halo of $A$ in $B$ and $s_{1}\left|W_{1}=s\right| W_{1}$. Clearly $W_{1} \cup U_{1}$ is a halo of $A \cup B_{1}=A_{1}$ in $B$. Proceeding inductively, we arrive at a sequence of sections $s_{n}: W_{n} \cup U_{n} \rightarrow E$, where $W_{n}$ is a halo of $A_{n}$ and such that $s_{n}\left|W_{n}=s_{n-1}\right| W_{n}$. We then define $S(x)$ $=s_{n}(x)$ if $x \in A_{n}$. By local finiteness, $S$ is continuous.

\section{BIBLIOGRAPHY}

1. J. Derwent, On the covering homotopy theorem, Nederl. A kad. Wetensch. Proc. Ser. A 62 (1959), 275-279.

2. A. Dold, Partitions of unity in the theory of fibrations, Ann. of Math. 78 (1963), 223-255.

3. J. L. Kelley, General topology, Van Nostrand, New York, 1955.

4. J. Milnor, Lectures on characteristic classes, notes by James Stasheff, Princeton Univ. Press, Princeton, N. J., 1957.

5. P. Holm, The microbundle representation theorem, Acta Math. 17 (1967), 191213.

University of Notre DAME 